\title{
Determining Motor Development Status of 3-10 Year- Old Children in Ahvaz City Using TGMD-3 Test
}

\author{
Farzad Mohammadi ${ }^{1}$, Abbas Bahram², Hasan Khalaji ${ }^{3 *}$, Farhad Ghadiri ${ }^{4}$ \\ ${ }^{1}$ PHD Student, Department of Motor Behavior, Faculty of Physical Education and Sport Sciences, Kharazmi University, \\ Tehran, Iran \\ ${ }^{2}$ Professor, Department of Motor Behavior, Faculty of Physical Education and Sport Sciences, Kharazmi University, Tehran, \\ Iran \\ ${ }^{3}$ Associate Professor, Department of Motor Behavior and Sport Psychology, Faculty of Sport Sciences, Arak University, Arak, \\ Iran \\ ${ }^{4}$ Assistant Professor, Department of Motor Behavior, Faculty of Physical Education and Sport Sciences, Kharazmi University, \\ Tehran, Iran
}

\section{*Correspondence to Hasan Khalaji, PHD; Associate Professor, Department of Motor Behavior and Sport Psychology, Faculty of Sport Sciences, Arak University, Arak, Iran. Tel: 989122054730; \\ Email: h-khalaji@araku.ac.ir}

\section{Received June 23, 2017} Accepted September 7, 2017 Published online September 31, 2017

\begin{abstract}
Introduction: The principle elements of motor development are fundamental movement skills (FMSs). The children with high levels of FMS are most likely to participate in health-related physical activities. This study aimed to determine motor development status of 3-10 year-old children in Ahvaz, Iran.

Methods: The research strategy and design were descriptive-normative and cross-sectional, respectively. A total of 1600 children aged 3-10 years were chosen from 8 regions of Ahvaz using cluster-sampling. The instrument used for data-collection was Test of Gross Motor Development - third edition (TGMD-3). In order to analyze the data, multivariate and Tukey post-hoc analyses were used along with Mann-Whitney $U$ and chi-square tests.

Results: Results showed that boys, compared to girls, had better levels of performance in locomotor skills of running, horizontal jump and sliding, while girls performed galloping and skipping better than boys. In all ball skills, boys had significant higher levels of performance compared to girls., Results also revealed that in both subtests, performance enhanced with age increase. Prevalence of low motor proficiency was observed in skipping, forehand strike and overhand throw, while there was prevalence of mastery in hoping, sliding, dribble and catching. Low motor proficiency was observed for several FMSs in girls.

Conclusion: Girls, compared to the boys of the same age, had lower levels of performance in all ball skills and some of locomotor skills. This result shows they are at the risk of unhealthy motor development; therefore, it is suggested that special programs and equal opportunities be prepared for them in order to enhance their motor competence in FMS.

Keywords: Fundamental movement skills, Gross motor development, Motor competence, Sex, Development delay
\end{abstract}

as follows: Mohammad F, Bahram A, Hasan Khalaji H, Ghadiri F. Determining motor development status of 3-10-year-old children in Ahvaz city using TGMD-3 test. Int J Basic Sci Med. 2017;2(3):139 146. doi:10.15171/ ijbms.2017.26. skills (FMSs) have been suggested as effective in children's tendency towards participation in physical activities.

FMSs are the principle elements of motor development ${ }^{5}$ and building blocks for complex and advanced movements. ${ }^{6}$ These skills are usually divided into object control skills and locomotor skills. ${ }^{7}$ Children with higher levels of FMS competence, are more likely to participate in physical activities. ${ }^{8}$ Research evidence has shown that obese and fat children have also less

Copyright $\odot 2017$ The Author(s); Published by Zabol University of Medical Sciences. This is an open-access article distributed under the terms of the Creative Commons Attribution License (http://creativecommons.org/licenses/by/4.0), which permits unrestricted use, distribution, and reproduction in any medium, provided the original work is properly cited. 
FMS competency. ${ }^{9,10}$ Thus, FMS development in children might play an important role in preventing childhood obesity.

Test of Gross Motor Development-third edition (TGMD3 ), as a modification of TGMD (1985) and TGMD-2 (2000), is one of the tests used to measure FMS in some countries. In this new version, object control skills are renamed as ball skills in order to provide better clarity. There have been also some changes in the criteria for skill performance; some skills have been either added or removed as well. ${ }^{11}$

Gender and age are among the FMS-affecting factors. Some gender-related studies have reported that boys have often higher levels of performance in FMS..$^{12-15}$ Some other studies have shown that boys are better at object control skills while girls have better locomotor skill proficiency. ${ }^{16,17}$ Yang et $\mathrm{al}^{18}$ and $\mathrm{Li}^{19}$ suggested that there was no significant difference between boys and girls in locomotor skills. Based on literature, the performance difference between girls and boys before puberty is not caused by their biological differences ${ }^{20}$; in fact, environmental factors might be considered as influential. There might be various reasons for the FMS performance differences between girls and boys, such as biased engagement in a specific sport, preferring physical activity and certain gender roles that have been defined for girls and boys. ${ }^{12}$ These factors are generally called socio-cultural factors which differ in every society; therefore, mixed results have been observed in terms of gender differences in FMS.

Age is also an important factor in FMS development. Yang et al showed that except for throwing and jumping skills, other locomotor and object control skills would steadily enhance from age 3 to $5 .{ }^{18}$ In another study, Spessato et al have suggested that performance enhances from age 5 to 7 and then, there is a flat performance from age 8 to $10 .^{21}$ Other researchers have shown that boys' scores increase with their age, while in girls, except for 5-8 year-old ones, performance enhances with their age increases. ${ }^{12}$

Today, many studies have been conducted all around the world on decreased FMS competence. Rosenberg et al showed that $13 \%$ of young children have delayed development. ${ }^{22}$ Considering the delayed FMS, Hardy et al necessitate intervention programs for FMS development in early childhood. ${ }^{23}$ Considering many studies suggesting the prevalence of low motor proficiency, it seems that low motor proficiency in children is a worldwide serious problem which might be the result of low physical activity and more tendency towards video games.

Due to the limited basic descriptive information about FMS in children living in Ahvaz city and mixed results offered by other studies based on gender and age differences in FMS, also, because of cultural differences as well as inter-family differences of motor development considering different cultural and geographical conditions in Ahvaz city (weather, air dust, etc.), it seems necessary to conduct such study. This study aimed to examine gross motor development status in 3-10 year-old children living in Ahvaz using TGMD-3. This study examined genderand age-based differences in FMS while describing the prevalence of mastery and low motor proficiency in FMS among Ahvazian children. The results of this study could help understand the process of estimating the motor development status of Ahvazian children while offering valid information for experts so that they can design and implement development programs in order to take an important step towards diagnosing children's delayed motor development and enhancing it.

\section{Methods}

Method and Design

The research strategy and design were descriptivenormative and cross-sectional, respectively. Data were collected using cross-sectional method during a specific period of time.

\section{Participants}

Participants included 1600 children aged 3-10 years (Mean age $=6.56 \pm 2.29$ years; $50 \%$ boy) living in Ahvaz, chosen by cluster sampling. Children with neurological and musculoskeletal problems were excluded from the study. We used their health records that were available at kindergartens or schools to diagnose musculoskeletal problems.

\section{Instruments}

TGMD-3 was used in order to measure the FMS in 3-10 year-old children with typical development. This test includes 6 locomotor skills (running, galloping, hoping, skipping, horizontal jump, and sliding) and 7 ball skills (2-hand strike of a stationary ball, one-hand forehand strike of self-bounced ball, one-hand stationary dribble, 2-hand catch, kicking a stationary ball, overhand throw, and underhand throw). In this test each skill was performed twice. In case of having a score, the examiner would score the skill as one, otherwise it would be zero. The examiner would sum up all the scores gained for 2 trials of performance for each criterion in order to obtain the raw score for every single skill. Each subtest had a raw score which was obtained by summing up the scores of all skills. The raw score for locomotor subtest was between 0 and 46, while the raw score for ball skills was between 0 and 54. The overall raw score for the test was between 0 and $100 .^{11}$

The construct validity was confirmed using confirmatory factor analysis. The intra-rater reliabilities of locomotor subscale, ball subscale, and the whole test were $\alpha=0.98$, $\alpha=0.99$, and $\alpha=0.99$, respectively. Moreover, in interrater reliability, intra-class correlation coefficients (ICC) for locomotor subscale, ball subscale, and the whole test were $\alpha=0.97, \alpha=0.98$, and $\alpha=0.98$, respectively. The internal consistency of the test was obtained using Cronbach $\alpha$ coefficients which were $\alpha=0.85, \alpha=0.85$, and 
$a=0.91$ for locomotor skill, ball skill, and the whole test. Results confirmed the validity and reliability of TGMD-3.

\section{Procedures}

In order to start the study, research permission was received from the Research Council for Ministry of Education and Welfare, Khuzestan province. Additionally, written consent from the parents of each child as well as his/her own consent were obtained for the participation in the research. After obtaining permission from authorities and applying ethical aspects in all stages of the study, participants were assessed by TGMD-3 in the selected kindergartens and primary schools. The evaluation protocol included presenting the correct performance of skill to children before starting the assessment. The child then would perform a practice trial after which 2 formal trials would be performed for each skill. Child's performance would also be recorded on a video camera which allowed the examiner to analyze the video after the test. Raw scores of each skill were analyzed in order to determine the low motor proficiency or mastery (adapted from Okely and Booth ${ }^{24}$ ) in each skill. Mastery was defined as the maximum scores or 1 score less than maximum in 2 trials. Low motor proficiency was defined as 0 and 1 score for both trials of each skill. For example, the 2-hand strike of a stationary ball included 5 performance criteria that must be demonstrated as:

5 criteria $* 2$ trials $=$ maximum score of 10 .

A child who scored 9 or 10 across 2 trials was placed in the high mastery category. While the child who earned a score of 0 or 1 , was considered in low motor proficiency category. In those skills with 4 criteria (e.g., running), the score of 7 or 8 would be considered as high mastery while the score of 0 or 1 would be considered as low motor proficiency.
Data Analysis

A multivariate analysis was used along with Tukey post hoc test in order to compare the gender- and agebased differences in raw scores of TGMD-3. Low motor proficiency and mastery were defined as a percentage of gender and age groups. Mann-Whitney $U$ and chi-square tests were used to compare low motor proficiency and mastery in terms of gender and age, respectively. Data were analyzed by SPSS version 22.0 with a significance level at $P<0.05$.

\section{Results}

Comparison of Performance Between Girls and Boys

Table 1 shows the mean and standard deviation of TGMD-3 raw scores in locomotor and ball skills between boys and girls. Multivariate analysis showed that there was a significant difference between boys' and girls' performance. (Wilks's $=0.80, \mathrm{~F}[13,1586]=28.8, P=$ 0.0001). The post hoc tests in Table 1 show that there was no significant difference between the total mean score of locomotor subtest of girls and boys, but the total mean score of boys' ball subtest was significantly higher than the girls'. Boys had better performance in locomotor skills of running, horizontal jump, and sliding, while girls were better in galloping and skipping. Although girls earned higher mean scores of hoping, the difference was not significant. Boys had significantly better performance in ball skills of 2-hand strike, one-hand forehand strike, stationary dribble, catching, kicking, overhand throw, and underhand throw.

Age-Based comparison

The results of multivariate analysis showed that there was a significant difference between the age groups of 3 to 10 (Wilks's $=0.13, \mathrm{~F}[91,9857.7]=41.7, P=0.0001$ ).

Table 1. Raw Scores of Locomotor and Ball Skills and Gender-Based Comparison

\begin{tabular}{|c|c|c|c|c|}
\hline \multirow[b]{2}{*}{ Subtest Skills } & \multicolumn{2}{|c|}{ Raw Score, Mean \pm SD } & \multirow[b]{2}{*}{$\boldsymbol{P}$} & \multirow[b]{2}{*}{$\eta 2$} \\
\hline & Girls & Boys & & \\
\hline Locomotor & $29.3 \pm 9.1$ & $29.2 \pm 9.1$ & 0.87 & 0.001 \\
\hline Run & $6.4 \pm 1.6$ & $6.7 \pm 1.6$ & $<0.0001$ & 0.01 \\
\hline Gallop & $4.6 \pm 1.8$ & $4.4 \pm 1.8$ & 0.008 & 0.004 \\
\hline Hop & $5.23 \pm 2.3$ & $5.08 \pm 2.6$ & 0.23 & 0.001 \\
\hline Skip & $3.03 \pm 2.3$ & $2.6 \pm 2.2$ & $<0.0001$ & 0.009 \\
\hline H-Jump & $3.26 \pm 1.8$ & $3.47 \pm 1.9$ & 0.025 & 0.003 \\
\hline Slide & $6.7 \pm 2.01$ & $6.9 \pm 1.7$ & 0.045 & 0.003 \\
\hline Ball skills & $24.2 \pm 9.4$ & $28.3 \pm 10.6$ & $<0.0001$ & 0.041 \\
\hline Two-hand strike & $4.8 \pm 2.07$ & $5.28 \pm 1.8$ & $<0.0001$ & 0.012 \\
\hline Forehand & $1.84 \pm 2.1$ & $2.5 \pm 2.2$ & $<0.0001$ & 0.027 \\
\hline Dribble & $2.9 \pm 1.9$ & $3.2 \pm 2.09$ & 0.008 & 0.004 \\
\hline Catch & $3.2 \pm 1.4$ & $3.5 \pm 1.6$ & 0.001 & 0.006 \\
\hline Kick & $3.7 \pm 1.7$ & $4.6 \pm 2.2$ & $<0.0001$ & 0.052 \\
\hline Overhand throw & $2.22 \pm 2.02$ & $3.21 \pm 2.09$ & $<0.0001$ & 0.055 \\
\hline Underhand throw & $5.3 \pm 1.9$ & $5.8 \pm 1.8$ & $<0.0001$ & 0.018 \\
\hline
\end{tabular}


Moreover, based on the results obtained from the age groups of 3 to 10 , there was a significant difference between total mean score of locomotor subtest $(P=$ $0.0001)$ and total mean score of ball skills subtest $(P=$ $0.0001)$. In locomotor skills, mean raw scores of running $(P=0.0001)$, galloping $(P=0.0001)$, hoping $(P=0.0001)$, skipping $(P=0.0001)$, horizontal jump $(P=0.0001)$ and sliding $(P=0.0001)$ were significantly different among different age groups of 3 to 10 (Figure 1).

In ball skills, the mean raw scores of 2-hand strike $(P=$ $0.0001)$, one-hand forehand strike $(P=0.0001)$, dribble $(P=0.0001)$, catching $(P=0.0001)$, kicking $(P=0.0001)$, overhand throw $(P=0.0001)$, and underhand throw $(P=$ 0.0001 ) were significantly different between different age groups of 3 to 10 (Figure 2).

Finally, Tukey post hoc test was used to compare the subtest performance of locomotor and ball skills between adjacent age groups (3-4, 4-5, 5-6, 6-7, 7-8, 8-9, 9-10). Results showed that total mean raw score of locomotor subtest significantly increased per year among 3 to 10 age groups $(P=0.0001)$.In locomotor skills, a significant increase was observed just in some skills as follows: running: between age groups of 3-4 $(P=0.0001), 4-5$ $(P=0.006)$, and 5-6 $(P=0.0001)$; galloping: between age groups of 4-5 $(P=0.035)$ and 5-6 $(P=0.0001)$; hoping: between age groups of 3-4 $(P=0.0001), 4-5(P=0.0001)$, 5-6 $(P=0.0001)$, 6-7 $(P=0.0001)$, and 8-9 $(P=0.007)$; skipping: between age groups of 3-4 $(P=0.0001), 4-5(P=$ $0.0001)$, 6-7 $(P=0.0001)$, and 8-9 $(P=0.004)$; horizontal jump: between age groups of 7-8 $(P=0.0001), 8-9(P=$ $0.004)$, and $9-10(P=0.001)$; and sliding: between age groups of 3-4 $(P=0.0001), 4-5(P=0.0001), 5-6 \quad(P=$ $0.002)$, and 6-7 $(P=0.036)$.

Based on the results, the total mean raw score of ball skills subtest significantly increased per year among age groups of 3 to $10(P=0.0001)$.

In ball skills, a significant increase was observed just in some skills as follows: 2-hand strike: between age groups of 3-4 $(P=0.0001), 5-6(P=0.0001), 8-9(P=0.004)$, and 9-10 $(P=0.041)$; forehand strike: between age groups of 4-5 $(P=0.0001)$, 5-6 years $(P=0.002), 6-7$ years $(P=$ $0.0001), 7-8$ years $(P=0.0001), 8-9$ years $(P=0.0001)$, and 9-10 years $(\mathrm{p}=0.002)$; dribble: between age groups of 3-4 $(P=0.043), 4-5(P=0.006), 6-7(P=0.0001), 7-8(P=$

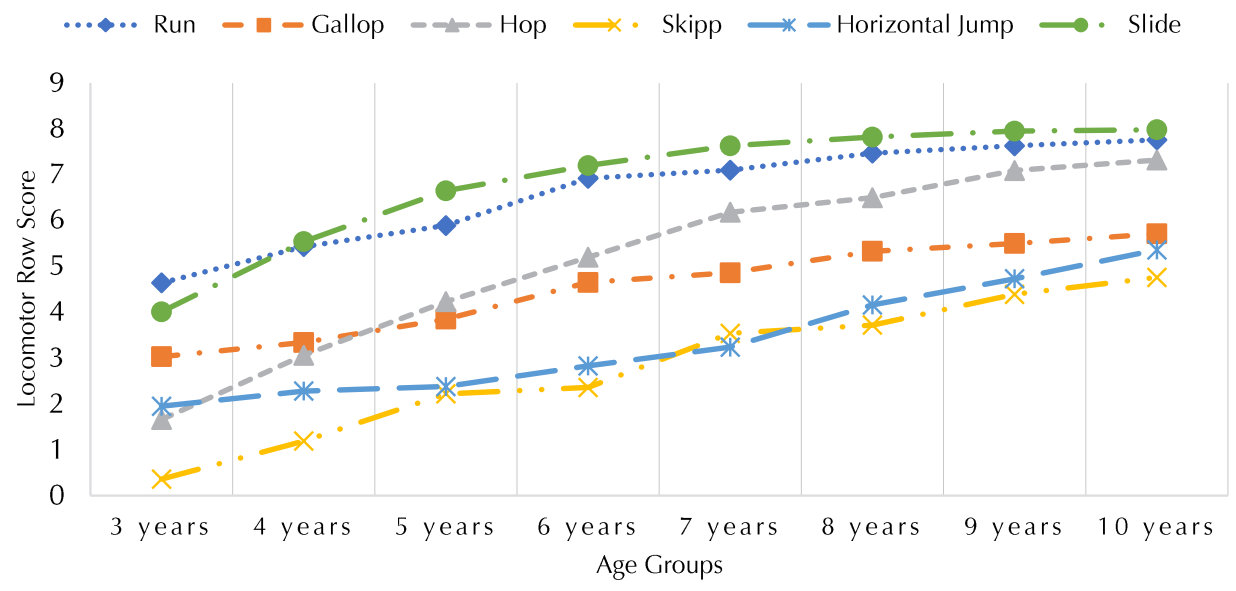

Figure 1. Mean Raw Scores of Locomotor Skills by Age Group.



Figure 2. Mean Raw Scores of Ball Skills by Age Group (Year). 
$0.0001)$, and $8-9$ years $(P=0.0001)$; catching: between age groups of 3-4 $(P=0.014), 4-5(P=0.0001), 6-7(P=$ $0.0001), 7-8(P=0.041)$, and $8-9(P=0.0001)$; kicking: between age groups of 3-4 $(P=0.002), 5-6(P=0.007)$, 6-7 $(P=0.0001)$, and 8-9 $(P=0.0001)$; over-hand throw: between age groups of 5-6 $(P=0.029)$; and under-hand throw: between age groups of 7-8 $(P=0.0001)$.

Gender-Based mastery and Low Motor Proficiency

Table 2 shows the frequency and relative frequency of mastery and low motor proficiency between girls and boys as well as gender-based comparison of the prevalence of proficiency in TGMD-3. In locomotor subtests, the prevalence of low motor proficiency was observed in skipping (boys: 38.3 and girls: 32.9 ). In ball skill subtests, the prevalence of low motor proficiency was observed in forehand strike (boys: 34.6 and girls: 50.5 ) and overhand throw (boys: 20.1 and girls: 37.3 ). The results of Mann-Whitney U test showed that in horizontal jump, dribble, and underhand throw, girls' level of lower motor proficiency was significantly higher than boys' $(P<0.05)$. The prevalence of mastery was observed in locomotor subtests of running (boys: 58.9, girls: 45.3), hoping (boys: 30.1, girls: 27.1) and sliding (boys: 67.9, girls: 65.3). In ball skill subtests, mastery was observed in boys' dribble (32.5) and catching (30.1). The results of Mann-Whitney $\mathrm{U}$ test showed that boys performed significantly better in motor skills compared to girls $(P<0.05)$. There was no significant difference between girls and boys in other skills $(P>0.05)$.

Age-Based Mastery and Low Motor Proficiency

Table 3 shows age-based frequency and relative frequency for the prevalence of low motor proficiency in locomotor and ball skills. Lower levels of motor proficiency were observed in some skills as follows: skipping in age groups of $3(89 \%)$ and $4(66.5 \%)$ years old; forehand strike in age groups of 3 (98\%), 4 (87.5\%), and 5 (59.5\%) years old; dribble in age group of 3 years old (56.5\%), and overhand throw in age group of 3 years old $(57.5 \%)$. The prevalence of low motor proficiency was less than $50 \%$ in other age groups and skills. The results of chi-square test showed that there was no significant difference between the 2 skills of 2-hand strike and underhand throw performed by age groups of 3 to 10 years old $(P>0.05)$. However, a significant age-based difference was observed in other locomotor and ball skills $(P<0.05)$. In general, the prevalence of low motor proficiency decreased with age increase.

Table 4 shows age-based frequency and relative frequency for the prevalence of mastery in locomotor and ball skills. Higher levels of mastery was observed in some skills as follows: running in age groups of $6(60.5 \%), 7$ (62\%), 8 (75.5\%), $9(83.5 \%)$, and $10(91.5 \%)$ years old; hoping in age groups of $9(62 \%)$ and $10(73 \%)$ years old; skipping in age group of 10 years old (59.5\%); sliding in age groups of 5 (52.5\%), 6 (73\%), 7 (86\%), 8 (94\%), 9 (98\%), and 10 (99\%) years old; dribble in age groups of $9(71 \%)$ and 10 (79\%) years old; catching in age groups of $9(69 \%)$ and $10(76.5 \%)$ years old; and underhand throw in age groups of $9(56 \%)$ and $10(66.5 \%)$ years old. The prevalence of mastery was lower than $50 \%$ in other age groups and skills.

The results of chi-square test showed significant agebased differences in all locomotor and ball skills $(P<0.05)$. In general, the prevalence of mastery increased

Table 2. Frequency and Relative Frequency of Low Motor Proficiency and Mastery in Locomotor and Ball Skills Subtests and Skills by Gender

\begin{tabular}{|c|c|c|c|c|c|c|c|c|}
\hline \multirow[b]{2}{*}{ Subtest Skills } & \multicolumn{3}{|c|}{ Low Proficiency, Frequency (Relative Frequency) } & \multirow[b]{2}{*}{$\boldsymbol{P}$} & \multicolumn{3}{|c|}{ Mastery, Frequency (Relative Frequency) } & \multirow[b]{2}{*}{$\boldsymbol{P}$} \\
\hline & $\begin{array}{c}\text { Boys } \\
\text { No. }(\%)\end{array}$ & $\begin{array}{c}\text { Girls } \\
\text { No. }(\%)\end{array}$ & $\begin{array}{c}\text { Total } \\
\text { No. }(\%)\end{array}$ & & $\begin{array}{c}\text { Boys } \\
\text { No. }(\%)\end{array}$ & $\begin{array}{c}\text { Girls } \\
\text { No. }(\%)\end{array}$ & $\begin{array}{c}\text { Total } \\
\text { No. }(\%)\end{array}$ & \\
\hline Locomotor & - & - & - & - & $3(0.4)$ & $5(0.6)$ & $8(0.5)$ & - \\
\hline Run & - & - & - & - & $471(58.9)$ & $362(45.3)$ & $833(52.1)$ & 0.170 \\
\hline Gallop & $73(9.1)$ & $67(8.4)$ & $140(8.8)$ & 1.00 & $23(2.9)$ & $40(5)$ & 63 (3.9) & 0.199 \\
\hline Hop & $102(12.8)$ & $69(8.6)$ & $171(10.7)$ & 0.625 & $241(30.1)$ & $217(27.1)$ & $458(28.6)$ & 0.400 \\
\hline Skip & $306(38.3)$ & $261(32.6)$ & $567(35.4)$ & 0.704 & $135(16.9)$ & $184(23)$ & 319 (19.9) & 0.612 \\
\hline H-Jump & $39(4.9)$ & $55(6.9)$ & $94(5.9)$ & 0.035 & $65(8.1)$ & $51(6.4)$ & $116(7.3)$ & 0.834 \\
\hline Slide & $9(1.1)$ & $10(1.3)$ & $19(1.2)$ & 0.968 & $543(67.9)$ & $522(65.3)$ & 1065 (66.6) & 0.138 \\
\hline Ball skills & - & - & - & - & - & - & - & - \\
\hline Two-hand strike & $2(0.3)$ & $18(2.3)$ & $20(1.3)$ & 0.126 & $24(3)$ & $23(2.9)$ & $47(2.9)$ & 0.057 \\
\hline Forehand & 277 (34.6) & $404(50.5)$ & $681(42.6)$ & 0.775 & $11(1.4)$ & $24(3)$ & $35(2.2)$ & 0.334 \\
\hline Dribble & $146(18.3)$ & $153(19.1)$ & 299 (18.7) & 0.050 & $260(32.5)$ & $193(24.1)$ & $453(60.2)$ & 0.917 \\
\hline Catch & $34(4.3)$ & $31(3.9)$ & $65(4.1)$ & 0.661 & $241(30.1)$ & $171(21.4)$ & $412(25.8)$ & 0.0001 \\
\hline Kick & $14(1.8)$ & $18(2.3)$ & $32(2)$ & 0.357 & $187(23.4)$ & $58(7.3)$ & $245(15.3)$ & 0.654 \\
\hline Overhand throw & $161(20.1)$ & $298(37.3)$ & $459(28.7)$ & 0.054 & $26(3.3)$ & $12(1.5)$ & $38(2.4)$ & 0.816 \\
\hline Underhand throw & $9(1.1)$ & $14(1.8)$ & $23(1.4)$ & 0.005 & 285 (35.6) & $220(27.5)$ & 505 (31.6) & 0.238 \\
\hline
\end{tabular}

Note: Mann-Whitney $U$ test, $P$ values for sex comparison. 
Table 3. Frequency and Relative Frequency of Low Motor Proficiency in Locomotor and Ball Skills Subtest and Skills by Age

\begin{tabular}{|c|c|c|c|c|c|c|c|c|c|}
\hline \multirow[b]{2}{*}{ Subtest Skill } & \multicolumn{8}{|c|}{ Frequency (Relative Frequency) } & \multirow[b]{2}{*}{$\boldsymbol{P}$} \\
\hline & $\begin{array}{c}3 \text { Years } \\
\text { No. }(\%)\end{array}$ & $\begin{array}{l}4 \text { Years } \\
\text { No. }(\%)\end{array}$ & $\begin{array}{l}5 \text { Years } \\
\text { No. }(\%)\end{array}$ & $\begin{array}{c}6 \text { Years } \\
\text { No. }(\%)\end{array}$ & $\begin{array}{c}7 \text { Years } \\
\text { No. }(\%)\end{array}$ & $\begin{array}{c}8 \text { Years } \\
\text { No. }(\%)\end{array}$ & $\begin{array}{c}9 \text { Years } \\
\text { No. }(\%)\end{array}$ & $\begin{array}{l}10 \text { Years } \\
\text { No. }(\%)\end{array}$ & \\
\hline Locomotor & - & - & - & - & - & - & - & - & - \\
\hline Run & - & - & - & - & - & - & - & - & - \\
\hline Gallop & $43(21.5)$ & $37(18.5)$ & $23(11.5)$ & $12(6)$ & $12(6)$ & $4(2)$ & $4(2)$ & $5(2.5)$ & $<0.0001$ \\
\hline Hop & $110(55)$ & $45(22.5)$ & $8(4)$ & $5(2.5)$ & $1(0.5)$ & - & - & $2(1)$ & $<0.0001$ \\
\hline Skip & $178(89)$ & $133(66.5)$ & $81(40.5)$ & $69(34.5)$ & $33(16.5)$ & $32(16)$ & $26(13)$ & $15(7.5)$ & $<0.0001$ \\
\hline $\mathrm{H}$-jump & $24(12)$ & $20(10)$ & $18(9)$ & $16(8)$ & $10(5)$ & $2(1)$ & $2(1)$ & $2(1)$ & $<0.0001$ \\
\hline Slide & $15(7.5)$ & $2(1)$ & $1(0.5)$ & - & - & $1(0.5)$ & - & - & $<0.0001$ \\
\hline Ball skills & - & - & - & - & - & - & - & - & $<0.0001$ \\
\hline Two-hand strike & $9(4.5)$ & $3(1.5)$ & - & - & - & $3(1.5)$ & $5(2.5)$ & - & 0.187 \\
\hline Forehand & $196(98)$ & $175(87.5)$ & 119 (59.5) & $81(40.5)$ & $54(27)$ & $29(14.5)$ & $13(6.5)$ & $14(7)$ & $<0.0001$ \\
\hline Dribble & $113(56.5)$ & 77 (38.5) & $42(21)$ & $33(16.5)$ & $17(8.5)$ & $9(4.5)$ & $4(2)$ & $4(2)$ & $<0.0001$ \\
\hline Catch & $29(14.5)$ & $15(7.5)$ & $10(5)$ & $5(2.5)$ & $1(0.5)$ & $5(2.5)$ & - & - & $<0.0001$ \\
\hline Kick & $18(9)$ & $1(0.5)$ & $4(2)$ & $2(1)$ & $5(2.5)$ & $1(0.5)$ & $1(0.5)$ & - & $<0.0001$ \\
\hline Over-hand throw & $115(57.5)$ & $91(45.5)$ & $68(34)$ & $59(29.5)$ & $36(18)$ & $33(16.5)$ & $38(19)$ & $19(9.5)$ & $<0.0001$ \\
\hline Under-hand throw & $10(5)$ & $5(2.5)$ & $3(1.5)$ & $3(1.5)$ & $2(1)$ & - & - & - & 0.062 \\
\hline
\end{tabular}

Note: Chi-square test, $P$ values for sex comparison.

Table 4. Frequency and Relative Frequency of Mastery in Locomotor and Ball Skills Subtest and Skills by Age

\begin{tabular}{|c|c|c|c|c|c|c|c|c|c|}
\hline \multirow[b]{2}{*}{ Subtest Skill } & \multicolumn{8}{|c|}{ Frequency (Relative Frequency) } & \multirow[b]{2}{*}{$\boldsymbol{P}$} \\
\hline & $\begin{array}{c}3 \text { Years } \\
\text { No. }(\%)\end{array}$ & $\begin{array}{c}4 \text { Years } \\
\text { No. }(\%)\end{array}$ & $\begin{array}{c}5 \text { Years } \\
\text { No. }(\%)\end{array}$ & $\begin{array}{c}6 \text { Years } \\
\text { No. }(\%)\end{array}$ & $\begin{array}{c}7 \text { Years } \\
\text { No. }(\%)\end{array}$ & $\begin{array}{c}8 \text { Years } \\
\text { No. }(\%)\end{array}$ & $\begin{array}{c}9 \text { Years } \\
\text { No. }(\%)\end{array}$ & $\begin{array}{l}10 \text { Years } \\
\text { No. }(\%)\end{array}$ & \\
\hline Locomotor & - & - & - & - & - & - & $3(1.5)$ & $5(2.5)$ & $<0.0001$ \\
\hline Run & $8(4)$ & $27(13.5)$ & $52(26)$ & $121(60.5)$ & $124(62)$ & $151(75.5)$ & $167(83.5)$ & $183(91.5)$ & $<0.0001$ \\
\hline Gallop & $1(0.5)$ & $2(1)$ & $1(0.5)$ & $4(2)$ & $2(1)$ & $7(3.5)$ & $18(9)$ & $28(14)$ & $<0.0001$ \\
\hline Hop & - & $1(0.5)$ & $4(2)$ & $29(14.5)$ & $67(33.5)$ & $87(43.5)$ & $124(62)$ & $146(73)$ & $<0.0001$ \\
\hline Skip & - & $2(1))$ & $10(5)$ & $8(4)$ & $36(18)$ & $48(24)$ & $96(48)$ & $119(59.5)$ & $<0.0001$ \\
\hline H-jump & - & - & - & $3(1.5)$ & $5(2.5)$ & $25(12.5)$ & $34(17)$ & $49(24.5)$ & $<0.0001$ \\
\hline Slide & $11(5.5)$ & $49(24.5)$ & $105(52.5)$ & $146(73)$ & $172(86)$ & $188(94)$ & $196(98)$ & $198(99)$ & $<0.0001$ \\
\hline Ball skills & - & - & - & - & - & - & - & - & $<0.0001$ \\
\hline Two-hand strike & $1(0.5)$ & - & $1(0.5)$ & $1(0.5)$ & $1(0.5)$ & $10(5)$ & $13(6.5)$ & $20(10)$ & $<0.0001$ \\
\hline Forehand & - & - & - & - & $4(2)$ & $4(2)$ & $11(5.5)$ & $16(8)$ & 0.008 \\
\hline Dribble & - & $2(1)$ & $10(5)$ & $21(10.5)$ & $38(19)$ & $82(41)$ & $142(71)$ & $158(79)$ & $<0.0001$ \\
\hline Catch & - & $2(1)$ & $10(5)$ & $18(9)$ & $37(18.5)$ & $54(27)$ & $138(69)$ & $153(76.5)$ & $<0.0001$ \\
\hline Kick & - & $1(0.5)$ & $1(0.5)$ & $6(3)$ & $40(20)$ & $40(20)$ & $77(38.5)$ & $80(40)$ & $<0.0001$ \\
\hline Over-hand throw & - & - & - & $1(0.5)$ & $4(2)$ & $4(2)$ & $13(6.5)$ & $16(8)$ & $<0.0001$ \\
\hline Under-hand throw & $14(7)$ & $19(9.5)$ & $22(11)$ & $46(23)$ & $65(32.5)$ & $94(47)$ & $112(56)$ & $133(66.5)$ & $<0.0001$ \\
\hline
\end{tabular}

Note: Chi-square test, $P$ values for sex comparison.

with age increase.

\section{Discussion}

The results of this study showed that there was no significant difference between total mean score of locomotor subtests for girls and boys, while boys' total mean score in ball skill subtests was significantly higher than girls'. Boys often presented better performance of locomotor skills such as running, horizontal jump, and sliding, while girls' level of performance was higher in galloping and skipping. Although girls had better hopping performance, the difference was not significant. These results are consistent with those reported by Spessato et al and Valentini et al who argued that boys had often better locomotor performance compared to girls, ${ }^{12,25}$ while the findings of the study do not match with those represented by Barnett et $\mathrm{al}^{17}$ and Hardy et al. ${ }^{16}$ The above-mentioned difference between the level of performance by girls and boys may be related to their gender-specific social roles. In Iran, some skills such as running and jumping, for example, are socially matched with boys' activities while some other skills like galloping, skipping, and hopping are commonly performed in young children's games. Additionally, parents allow boys to participate in challenging high-contact games home outside, while prohibiting girls from engaging in such games, thereby giving them no motivation, encouragement, support, and equal opportunity (7). In ball skill subtests, boys had better performance in all skills compared to girls. This is consistent with the results reported by Barnett et al, 
Hardy et al, Okely and Booth, and Valentini et al. 16,17,24,26 Considering the fact that in our society (Iran), girls are given no opportunity of sports activities more than their participation in physical education classes, their weak performance in ball skills requiring equipment is expected; especially when it comes to some specific skills such as "hand strike to a stationary ball" used in sports like baseball which are not part of our sport culture in Iran. Unfortunately, there were no equipment such as tennis rocket and ball, baseball bat and age-appropriate basketballs in more than $90 \%$ of the studied schools. The situation was much worse in surveys conducted in kindergartens. Most kindergartens in Ahvaz lack open space for physical and motor activities. Furthermore, there is no physical education teacher and motor development expert in the kindergartens. It seems that having certified physical education teachers and motor development experts is not legally mandatory for kindergartens. Unfortunately, most of those in kindergartens who deal with children only care about children's cognitive development and rather engage in activities such as painting.

The comparison of performance between adjacent age groups showed that with age increase, a significant increase was observed in locomotor skills as follows: running: from 3 to 6; galloping: from 4 to 6; hopping: from 3 to 9; skipping: from 3 to 5 and 6 to 9; horizontal jump: from 7 to 10, and sliding: from 3 to 7 . Results also showed that with age increase, a significant increase was observed in ball skills as follows: 2-hand strike: from 3 to 4,5 to 6 , and 8 to 10 ; forehand strike: from 4 to 10 ; dribble: from 3 to 5 and 6 to 9; catching: from 3 to 5 and 6 to 9; kicking: from 3 to 4,5 to 7 , and 8 to 9; overhand throw: from 5 to 6; and underhand throw: from 7 to 8 . These results are consistent with those reported by Yang et al, Li, and Spessato et al which showed that FMS tends to improve with age increase. ${ }^{18,19,21}$ The difference between children's performance before puberty could be rather due to the environmental factors such as practice, encouragement, and instruction. As the child grows older, there would be more opportunities in physical activities through training and experience. Moreover, as the child grows older, his/ her strength and physical capacity improves which in turn influences his/her motor performance.

Based on the results, the prevalence of low motor proficiency was observed in skipping (boys: 38.3, girls: 32.9), forehand strike (boys: 34.6, girls: 50.5), and overhand throw (boys: 20.1, girls: 37.3 ). This might be due to the fact that skipping has the most complicated movement pattern among locomotor skills and, also, as long as the child's neuromuscular system is not able to coordinate the movements of the leg (each having its own complicated pattern), skipping pattern may not be observed.7 The children presented low motor proficiency in forehand strike among ball skill subtests which might be due to the fact that this skill is not common in physical educational programs of Iranian schools, leading to children's lack of prior experience and familiarity with its performance criteria. This skill also requires the child's familiarity with the principles of biomechanics such as open kinetic chain and differentiated rotation of trunk at different times at which the children are not expected to be skillful at an early age. Results also revealed that girls had lower motor proficiency in horizontal jump, dribble, and underhand throw, compared to boys. This could be due to the social expectations. Girls' traditional games and activities are generally non-competitive including simple rhythmic and equilibrium movements such as skipping, galloping, hopping, and jumping rope. ${ }^{7}$ These non-complicated non-competitive games do not provide enough opportunity for them in order to engage in more difficult and competitive sports such as throwing and jumping.

The prevalence of mastery was observed in locomotor subtests of sliding (boys: 67.9, girls: 65.3), running (boys: 58.9, girls: 45.3), and hopping (boys: 30.1, girls: 27.1). A significant difference was observed just in the following skills and age groups: running in age group of 3 to 6 years old and sliding in age group of 3 to 7 years old; this could be due to the fact that the ceiling of performance for these 2 skills is reached at younger ages. Ulrich observed running and sliding as the skills which get mature before other skills. ${ }^{27}$ In general, the prevalence of low motor proficiency decreased while mastery increased with age increase.

The research limitations included a lack of information on patterns of nutrition and daily activity, differences in socio-economic status, genetic characteristics, and individual differences among participants. It is suggested that future studies examine these variables. Other research limitation in our study was the research strategy. Crosssectional design was used in this study and data were collected for a certain period of time. In cross-sectional design, it is possible to observe the difference but not the change. It is suggested that later studies be carried out using longitudinal design in order to examine the motor development of Ahvaz children and to provide a more accurate analysis.

\section{Conclusion}

Competency of FMS is a prerequisite for children's participation in different physical activities and games; therefore, its development and improvement is closely related to the child's cognitive, emotional, and psychomotor development. Thus, it is important to study the motor development status of children. The results of this study confirmed the developing nature of FMS, as the performance by age groups of 3 to 10 showed a significant improvement. Moreover, low motor proficiency was observed for several FMSs in girls. Considering the fact 
that the sampled girls showed lower levels of performance in all ball skills and some locomotor skills compared to the boys, it could be concluded that they are at risk of motor delay and unhealthy motor development. Therefore, it is suggested that their motor competence of FMSs be improved through appropriate developmental programs as well as providing equal opportunities for their participation in physical activities.

\section{Ethical Approval}

Local Ethics Committee of Sport Sciences College at the University of Kharazmi approved the study protocol as a master proposal in 2016.

\section{Competing Interests}

The authors declare no conflict of interests.

\section{Acknowledgements}

This research was carried out in cooperation with Ahvaz Ministry of Education. We thank all the research participants, school administrators, and physical education teachers who were selected for the research and helped us with the implementation of research protocol. It is worth mentioning that this paper was extracted from $\mathrm{PhD}$ dissertation, which was funded by Kharazmi University of Tehran.

\section{References}

1. Tsai CY, Yang SC. Study on the appearance of childhood games. J Educ Stud. 2012;46(1):1-19.

2. Swinburn BA, Sacks G, Hall KD, et al. The global obesity pandemic: shaped by global drivers and local environments. Lancet. 2011;378(9793):804-814. doi:10.1016/s01406736(11)60813-1.

3. Sullivan DA. Environmental factors in obesity. In: Nriagu J, ed. Encyclopedia of Environmental Health. Elsevier; 2011:380-391.

4. Malina RM, Bouchard C, Bar-Or O. Growth, Maturation, and Physical Activity. 2nd ed. Champaign, IL: Human Kinetics; 2004.

5. Reeves L, Broeder CE, Kennedy-Honeycutt L, East C, Matney L. Relationship of fitness and gross motor skills for five- to six-yr.-old children. Percept Mot Skills. 1999;89(3 Pt 1):739-747. doi:10.2466/pms.1999.89.3.739

6. Clark JE, Metcalfe JS. The mountain of motor development: A metaphor. Motor Development: Research and Reviews. 2002;2:163-190.

7. Haywood K, Getchell N. Life Span Motor Development. 6th ed. Champaign, IL: Human Kinetics; 2014.

8. Kim S, Kim MJ, Valentini NC, Clark JE. Validity and reliability of the TGMD-2 for South Korean children. J Mot Behav. 2014;46(5):351-356. doi:10.1080/00222895.2014.91 4886.

9. Logan SW, Getchell N. The relationship between motor skill proficiency and body mass index in children with and without dyslexia: a pilot study. Res Q Exerc Sport. 2010;81(4):518-523. doi:10.1080/02701367.2010.1059971 3.

10. Logan SW, Scrabis-Fletcher K, Modlesky C, Getchell N. The relationship between motor skill proficiency and body mass index in preschool children. Res Q Exerc Sport.
2011;82(3):442-448. doi:10.1080/02701367.2011.1059977 6.

11. Ulrich DA. Test of Gross Motor Development. 3rd ed. Austin TX: Pro-Ed; 2016.

12. Spessato BC, Gabbard C, Valentini N, Rudisill M. Gender differences in Brazilian children's fundamental movement skill performance. Early Child Dev Care. 2013;183(7):91623. doi:10.1080/03004430.2012.689761.

13. Ka Yee Wong A, Cheung SY. Gross Motor Skills Performance of Hong Kong Chinese Children. Journal of Physical Education Recreation. 2006;12(2):23.

14. Griffiths G, Billard R. The fundamental movement skills of a year 9 group and a gifted and talented cohort. Advances in Physical Education. 2013;3(4):215-220. doi: 10.4236/ ape.2013.34035.

15. Goodway JD, Robinson LE, Crowe H. Gender differences in fundamental motor skill development in disadvantaged preschoolers from two geographical regions. Res Q Exerc Sport. 2010;81(1):17-24.

16. Hardy LL, King L, Farrell L, Macniven $\mathrm{R}$, Howlett S. Fundamental movement skills among Australian preschool children. J Sci Med Sport. 2010;13(5):503-508. doi:10.1016/j.jsams.2009.05.010.

17. Barnett LM, van Beurden E, Morgan PJ, Brooks LO, Beard JR. Childhood motor skill proficiency as a predictor of adolescent physical activity. J Adolesc Health. 2009;44(3):252-259. doi:10.1016/j.jadohealth.2008.07.004.

18. Yang SC, Lin SJ, Tsai CY. Comparison of fundamental movement skills among young children with different gender, age, and BMI. Sports Exercise Research. 2014;16(3):287-296. doi: 10.5297/ser.1603.003.

19. Li J. Children's gross motor development from ages 3 to 10 in Shandong. Journal of Shandong Institute of Physical Education and Sports. 2009;25(4):47-50.

20. Gabbard CP. Lifelong motor development. San Francisco: Pearson Higher Ed; 2011.

21. Spessato BC, Gabbard C, Valentini NC. The role of motor competence and body mass index in children's activity levels in physical education classes. J Teach Phys Educ. 2013;32(2):118-130. doi: 10.1123/jtpe.32.2.118.

22. Rosenberg SA, Zhang D, Robinson CC. Prevalence of developmental delays and participation in early intervention services for young children. Pediatrics. 2008;121(6):e1503-1509. doi:10.1542/peds.2007-1680.

23. Hardy LL, Reinten-Reynolds T, Espinel P, Zask A, Okely AD. Prevalence and correlates of low fundamental movement skill competency in children. Pediatrics. 2012;130(2):e390398. doi:10.1542/peds.2012-0345.

24. Okely AD, Booth ML. Mastery of fundamental movement skills among children in New South Wales: prevalence and sociodemographic distribution. J Sci Med Sport. 2004;7(3):358-372.

25. Valentini NC, Logan SW, Spessato BC, de Souza MS, Pereira KG, Rudisill ME. Fundamental motor skills across childhood: age, sex, and competence outcomes of Brazilian children. J Mot Learn Dev. 2016;4(1):16-36. doi: 10.1123/ jmld.2015-0021.

26. Valentini NC, Spessato BC, Rudisill ME. Fundamental motor skills: a description of the most common errors demonstrated by children. J Sport Exerc Psychol. 2007;29:47-48.

27. Ulrich DA. Test of gross motor development. Austin, TX: PRO-ED Inc; 2000 